\title{
microRNA-9 might be a novel protective factor for osteoarthritis patients
}

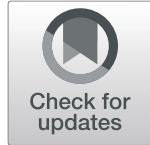

Lei Jiang, Xu Sun and Hongyang Kong*

\begin{abstract}
Background: The study aimed to identify the targeting genes and miRNAs using the microarray expression profile dataset for Osteoarthritis (OA) patients. Differentially expressed genes (DEGs) between OA and control samples were identified using Bayes method of limma package. Subsequently, a protein-protein interaction (PPI) network was constructed. miRNAs and transcription factor (TFs) based on DEGs in PPI network were identified using Webgestalt and ENCODE, respectively. Finally, MCODE, Gene Ontology (GO) function, and Kyoto Encyclopedia of Genes and Genomes (KEGG) were performed. The expressions of several DEGs and predicted miRNAs in OA rats were detected by RT-PCR.

Results: A total of 594 DEGs were identified. In PPI network, there were 313 upregulated DEGs and 22 downregulated DEGs. Besides, the regulatory relationships included 467 upregulated interactions and 85 downregulated interactions (miR-124A $\rightarrow$ QKI and MAP 1B) between miRNA and DEGs in PPI network. The module from downregulated DEGs-TFs-miRNA networks was mainly enriched to low-density lipoprotein particle clearance, response to linoleic acid, and small molecule metabolic process BP terms. Moreover, QKI, MAP TB mRNA and miR-9 expressions were significantly reduced in OA rats.

Conclusion: miR-9 might be a protective factor for OA patients via inhibiting proliferation and differentiation of cartilage progenitor cells. miR-124A might play an important role in progression of OA through targeting QKI and MAP $1 B$.
\end{abstract}

Keywords: Osteoarthritis, microRNAs, Protein-protein interaction, Modules, protective factor

\section{Background}

Osteoarthritis (OA) is the most prevalent rheumatic disease with the characteristics of chronic, debilitating and degenerative disease of the joints [1]. Approximately $1 / 3$ of OA patients is over 60 years old [2]. Besides, OA often occurs in people aged 60-70 years, more frequently in females (18\%) than males (10\%) [1] . Currently, treatments are only used to manage symptoms due to complex etiology [3]. Although some pathogenesis pathways have been reported, current study is incomplete for prevention or treatment. These limitations might be

\footnotetext{
*Correspondence: konghongyang1991@163.com

Department of Orthopedics, Taizhou People's Hospital, No. 366 Taihu Road, Taizhou City 225300, Jiangsu Province, China
}

surmounted through investigating the molecular mechanisms in OA.

microRNAs (miRNAs), as novel regulators of gene expression, play key roles in biological processes of OA [4]. Previous study has found that some miRNAs associates with musculoskeletal system [5-7]. miR-140 is involved in the cartilage of mouse embryos during the development of long and flat bone through directly targeting distone deacetylase 4 (HDAC4) [8]. In addition, miR-140 protects against OA development by targeting ADAM metallopeptidase with thrombospondin type 1 motif 5 (Adamts-5) expression [9]. The tumor necrosis factor a (TNF- $\alpha)$ is an important inflammatory factor, and its expression level is increased in OA synovial membranes [10]. Decreased expression of miR-130a,

(c) The Author(s). 2020 Open Access This article is licensed under a Creative Commons Attribution 4.0 International License, which permits use, sharing, adaptation, distribution and reproduction in any medium or format, as long as you give appropriate credit to the original author(s) and the source, provide a link to the Creative Commons licence, and indicate if changes were made. The images or other third party material in this article are included in the article's Creative Commons licence, unless indicated otherwise in a credit line to the material. If material is not included in the article's Creative Commons licence and your intended use is not permitted by statutory regulation or exceeds the permitted use, you will need to obtain permission directly from the copyright holder. To view a copy of this licence, visit http://creativecommons.org/licenses/by/4.0/ The Creative Commons Public Domain Dedication waiver (http://creativecommons.org/publicdomain/zero/1.0/) applies to the data made available in this article, unless otherwise stated in a credit line to the data. 
correlated with TNF- $\alpha$ in the development of OA [6]. Thus, miR-140 and miR-130a are novel therapeutic targets in OA development. Therefore, it is necessary to identify more miRNAs associated with OA development in the future.

The present study aimed to identify the targeting genes and miRNAs using the microarray expression profile dataset. The differentially expressed genes (DEGs) were identified using Bayes method of limma package. Subsequently, a protein-protein interaction (PPI) network for these DEGs was constructed. miRNAs and transcription factor (TFs) based on DEGs in PPI network were identified using Webgestalt and ENCODE, respectively. Finally, significant network modules were obtained by MCODE, and their Gene Ontology (GO) function and Kyoto Encyclopedia of Genes and Genomes (KEGG) were enriched.

\section{Results}

The results of DEGs involved in the function and pathway for $\mathrm{OA}$

Totally, 594 DEGs were identified between OA and control samples, including 535 upregulated DEGs (MMP2, $F O S$, et al) and 59 downregulated DEGs (QKI, MAPB1, et al). The up-regulated DEGs were enriched to $1166 \mathrm{BP}$ terms, 20 KEGG pathways, and 83 Reactome pathways. The top 5 of these were showed in Table 1. The down-

Table 1 The top 5 of biological processes (BP) terms, Kyoto Encyclopedia of Genes and Genomes (KEGG) pathways, and Reactome pathways for upregulated differentially expressed genes (DEGs)

\begin{tabular}{ll}
\hline GO_ID & BP term Pathway \\
GO:0030198 & extracellular matrix organization \\
GO:0009888 & tissue development \\
GO:0045597 & positive regulation of cell differentiation, \\
GO:0007155 & cell adhesion \\
GO:0022610 & biological adhesion \\
KEGG_ID & KEGG Pathway \\
04350 & TGF-beta signaling pathway \\
04510 & Focal adhesion \\
04142 & Lysosome \\
04512 & ECM-receptor interaction \\
05110 & Vibrio cholerae infection \\
Reactome_ID & Reactome Pathway \\
$1,442,490$ & Collagen degradation \\
$3,000,170$ & Syndecan interactions \\
216,083 & Integrin cell surface interactions \\
$1,474,228$ & Degradation of the extracellular matrix \\
$1,474,244$ & Extracellular matrix organization \\
\hline
\end{tabular}

regulated DEGs were enriched to $480 \mathrm{BP}$ terms, 4 KEGG pathways, and 27 Reactome pathways. The top 5 of these were showed in Table 2.

\section{The DEGs-TFs-miRNA integrated network}

In PPI network, there were 313 upregulated DEGs with 841 interactions and 22 downregulated DEGs with 31 interactions (Fig. 1). Among them, 25 upregulated DEGs (GLIS2, FOSB, et al) and one downregulated DEG (MYF6) were also TFs, which have a transcriptional regulatory relationship with other DEGs. In addition, the regulatory relationships between miRNA and DEGs in PPI network were also identified (e.g. miR-124A $\rightarrow$ QKI and MAP 1B), including 467 upregulated interactions and 85 downregulated interactions. The DEGs-TFs-miRNA networks based on upregulated and downregulated DEGs are showed in Figs. 2 and 3, respectively. The nodes with degree more than 20 in upregulated DEGs-TFs-miRNA network are showed in Table 3, which presents 17 miRNAs (eg., miRNA-9) and 9 TFs. In addition, in the downregulated DEGs-TFs-miRNA network, QKI had the largest degree value (degree $=21$ ) and only one MYF6 TF was predicted. There were 4 miRNAs (eg., miR-124A) and 11 DEGs (eg., QKI and MAP $1 B$ ) in top 15 nodes listed by degree (Table 4), and QKI and MAP 1B were both predicted as target of miR-124A.

Table 2 The top 5 of BP terms, KEGG pathways, and Reactome pathways for downregulated DEGs

\begin{tabular}{ll}
\hline GO_ID & BP term Pathway \\
GO:0034439 & lipoprotein lipid oxidation \\
GO:0006629 & lipid metabolic process \\
GO:0044281 & small molecule metabolic process \\
GO:0010876 & lipid localization \\
GO:0044255 & cellular lipid metabolic process \\
KEGG_ID & KEGG Pathway \\
00982 & Drug metabolism - cytochrome P450 \\
04975 & Fat digestion and absorption \\
04920 & Adipocytokine signaling pathway \\
03320 & PPAR signaling pathway \\
Reactome_ & Reactome Pathway \\
ID & \\
75,109 & Triglyceride Biosynthesis \\
163,560 & Hormone-sensitivelipase (HSL)-mediated triacylglycerol \\
535,734 & hydrolysis \\
$1,266,738$ & Fatty acid, triacylglycerol, and ketone body metabolism \\
381,340 & Developmental Biology, \\
& Transcriptional regulation of white adipocyte \\
\hline & differentiation \\
\hline
\end{tabular}




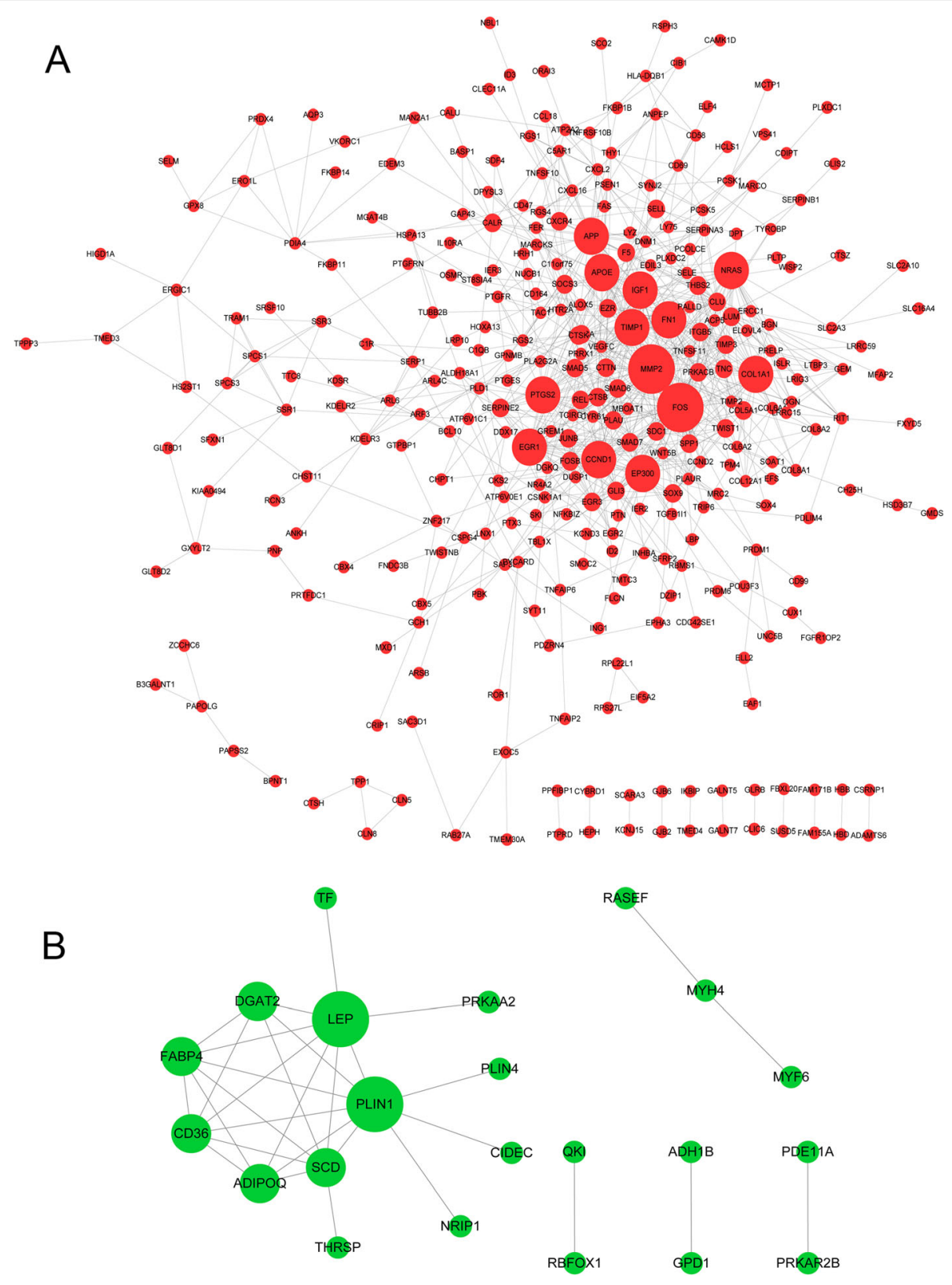

Fig. 1 The protein-protein interaction (PPI) network for upregulated differentially expressed genes (DEGs) (a) and downregulated DEGs (b). Red nodes indicate upregulated DEGs, green nodes indicate downregulated DEGs, and the black lines indicate the interactions. The node is larger, and it degree is higher, suggesting that it has more interactions with other proteins in PPI network

\section{The significant network modules in the integrated network}

Overall, 12 modules were identified from the upregulated network. The module with the highest Mcode score (5.739) included 24 nodes and 66 interactions (Fig. 4a). These DEGs in this module were mainly enriched to extracellular matrix organization, extracellular matrix disassembly, and collagen catabolic process BP terms, as well as ECM-receptor interaction, focal adhesion, and PI3K-Akt signaling pathways. Moreover, only one module was obtained from the downregulated network (Mcode score $=6.667$ ), including 7 nodes and 20 interactions (Fig. 4b). They were mainly enriched to low-density lipoprotein particle clearance, response to linoleic acid, and small molecule metabolic process BP terms.

\section{The results of qRT-PCR}

As shown in Fig. 5, expressions of QKI Mrna $(P<0.01)$ and miR-9 $(P<0.05)$ were significantly reduced in OA 


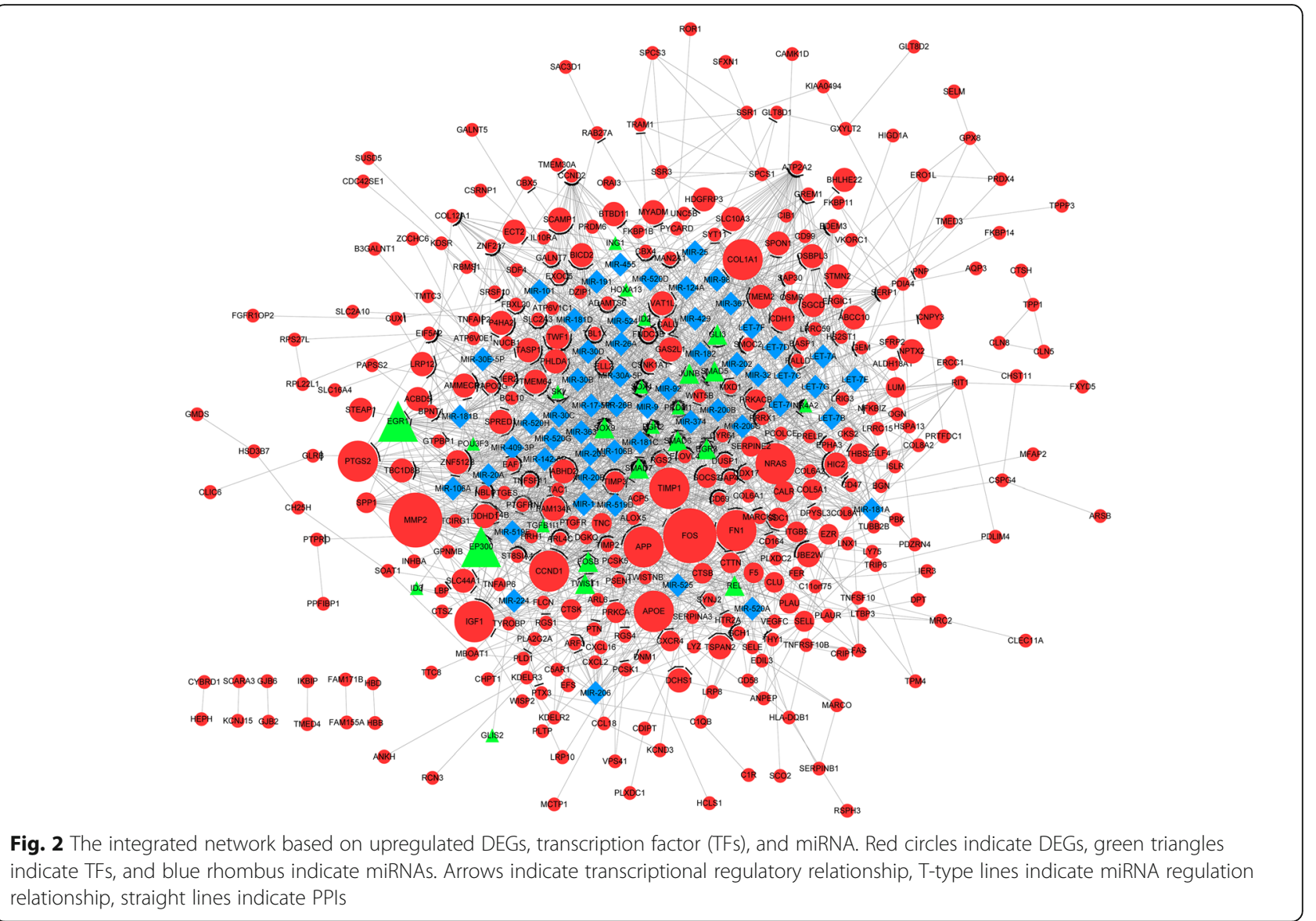

rats. Although expression of $M A P 1 B$ was also reduced in synovial tissue of OA rats, there were no significant differences when compared with that of health rats.

\section{Discussion}

In this study, 535 upregulated DEGs and 59 downregulated DEGs (e.g. QKI and MAPB1) were identified between $\mathrm{OA}$ and control samples. Among them, 313 upregulated DEGs with 841 interactions and 22 downregulated DEGs with 31 interactions were used to construct PPI network. As a result, 25 upregulated DEGs and one downregulated DEG in PPI network having a transcriptional regulatory relationship with other DEGs were obtained. In upregulated DEGs-TFs-miRNA network, miR-9 had higher degree values and was predicted to regulate 22 targets. Besides, the regulatory relationships (e.g. miR-124A $\rightarrow Q K I$ and $M A P \quad 1 B$ ) were highlighted in downregulated DEGs-TFs-miRNA network, in which $Q K I$ had the largest degree value, and QKI and MAP $1 B$ in top 15 nodes were both predicted as target of miR-124A. The module from downregulated DEGs-TFs-miRNA networks was mainly enriched to low-density lipoprotein particle clearance, response to linoleic acid, and small molecule metabolic process BP terms. Moreover, it was confirmed that expressions of QKI, MAP $1 B$ mRNA and miR-9 were significantly reduced in OA rats.

As is described above, miRNAs are novel regulators of gene expression and play key roles in biological processes of OA. miR-9 expression is upregulated in osteosarcoma tissues when compared with human osteoblastic hFOB cells and the corresponding noncancerous bone tissues [11]. Whereas osteosarcoma is derived from progenitor cells, which can be differentiated into bone, cartilage and fiber [12]. In other word, cartilage contain a number of stem cells or progenitor cells, which has been confirmed by a previous study [13]. In addition, Jiang et al. have found that cartilagederived stem/progenitor cells might have a key role in cartilage repair, which will behave therapeutic potential in OA [14]. MiR-9 has been identified to play critical roles in proliferation and differentiation of retinal progenitor cell and neural progenitor cells $[15,16]$. In a round-about way, miR-9 might play a consequence role in $\mathrm{OA}$ through regulating proliferation and differentiation of cartilage progenitor cell for cartilage repair. In the present study, miR-9 expression is reduced in OA rats when compared with the healthy rats, suggesting 


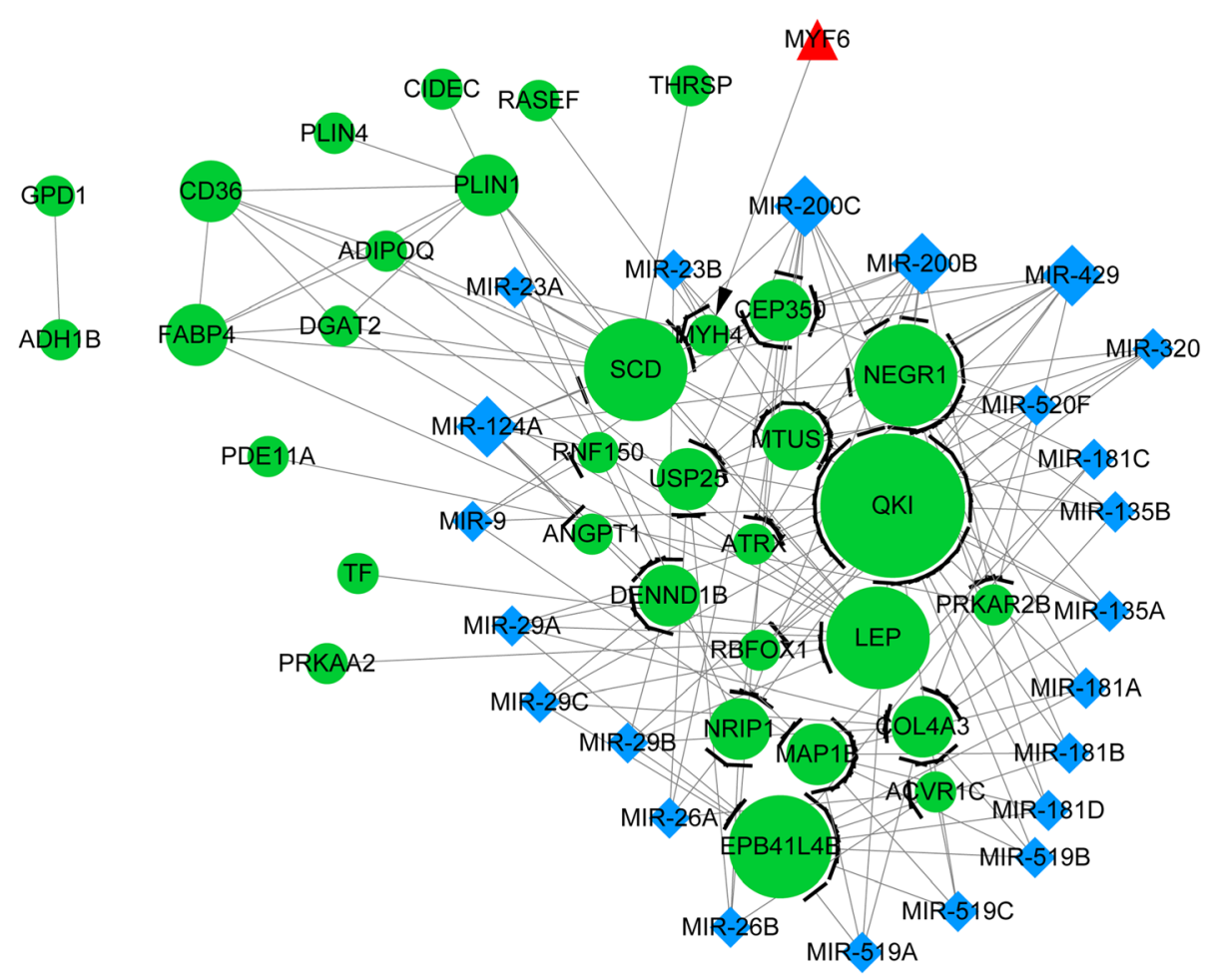

Fig. 3 The integrated network based on downregulated DEGs, transcription factor (TFs), and miRNA. Green circles indicate DEGs, red triangles indicate TFs, and blue rhombus indicate miRNAs. Arrows indicate transcriptional regulatory relationship, T-type lines indicate miRNA regulation relationship, straight lines indicate PPIs

miR-9 is involved in pathological process of OA. Similarly, a previous study indicates that expression level of miR-9 is decreased in OA chondrocytes with numerous apoptotic cell deaths through upregulating PRTG [17]. Therefore, miR-9 might be a novel protective factor for OA patients via inhibiting proliferation and differentiation of cartilage progenitor cells.

In the present study, KH Domain Containing RNA Binding (QKI) and Microtubule Associated Protein 1B (MAP 1B) were predicted as target genes of miR-124A. miR-124 plays roles in various pathologic conditions such as inflammatory responses, and osteoclast differentiation [18] miR-124A is overexpressed in OA cartilage with cocultured chondrocytes derived from MSC [19, 20]. Notably, it shows the alterations in chondrogenesis and neurogenesis are associated with the development of OA [21]. QKI belongs to a family of RNA-binding proteins called STAR proteins for Signal Transduction and Activation of RNA [22]. Recently, a study has confirmed that $Q K I$ genes is significantly suppressed when synovial explants were treated with miR-27b-3p in OA patients [23]. MAP $1 B$ belongs to the microtubule-associated protein family and is involved in microtubule assembly [24]. Actin cytoskeleton and microtubule array play essential roles on chondrogenesis [25]. It has been reported that expression of $M A P 1 B$ in mRNA and protein levels is significantly upregulated in degraded cartilage by Steinberg [26]. The 3'UTR of MAP $1 B$ mRNA interacts with QKI resulted in reducing MAP $1 B$ mRNA expression in the QKI-deficiency mutant mice. Meanwhile, RNAi-mediated QKI-knockdown caused destabilization of the MAP $1 B$ mRNA in oligodendroglia CG4 cell line [27]. Furthermore, overexpression of exogenous $Q K I$ was able to facilitate $M A P 1 B$ expression. Interesting, $Q K I$ and MAP $1 B$ mRNA expression levels were also reduced in rats with $\mathrm{OA}$ in this study. Therefore, we inferred miR-124A might play an important role in progression of OA through targeting QKI and MAP $1 B$.

However, there are still some limitations in the study. All of miRNAs in DEGs-TFs-miRNAs were predicted in OA samples using bioinformatic analysis, and their expressions and regulatory relationships should be verified using future experiments. In addition, these results will be confirmed using human samples. Moreover, the expression of MAP $1 B$ was reduced in synovial tissue of OA rats than in health rats, but no significant differences, which should be verified in large samples in future.

\section{Conclusion}

miR-9 might be a novel protective factor for OA patients OA synovia via inhibiting proliferation and differentiation 
Table 3 The nodes with degree more than 20 in the integrated network based on upregulated DEGs, transcription factor (TFs), and miRNA. The blod fonts indicate miRNA, the italic indicate TFs

\begin{tabular}{|c|c|c|c|}
\hline Symbol & Degree & Symbol & Degree \\
\hline FOS & 51.0 & MIR-30D & 27.0 \\
\hline COL1A1 & 36.0 & MIR-30C & 27.0 \\
\hline CCND1 & 36.0 & MIR-30A-5P & 27.0 \\
\hline ATP2A2 & 35.0 & SMAD6 & 26.0 \\
\hline APP & 35.0 & TIMP1 & 25.0 \\
\hline MMP2 & 34.0 & PTGS2 & 25.0 \\
\hline NRAS & 34.0 & $\mathrm{HIC2}$ & 25.0 \\
\hline FN1 & 34.0 & FNDC3B & 25.0 \\
\hline SMAD7 & 34.0 & TIMP3 & 24.0 \\
\hline EGR3 & 33.0 & PRRX1 & 24.0 \\
\hline MIR-519D & 32.0 & SOX9 & 23.0 \\
\hline MIR-20B & 32.0 & MIR-524 & 23.0 \\
\hline MIR-106B & 32.0 & MIR-124A & 22.0 \\
\hline MIR-106A & 32.0 & GLI3 & 22.0 \\
\hline MIR-20A & 32.0 & MIR-9 & 22.0 \\
\hline MIR-17-5P & 32.0 & CCND2 & 22.0 \\
\hline EGR1 & 30.0 & MIR-429 & 21.0 \\
\hline IGF1 & 29.0 & MIR-200C & 21.0 \\
\hline NR4A2 & 29.0 & MIR-200B & 21.0 \\
\hline EP300 & 28.0 & PRKACB & 21.0 \\
\hline APOE & 27.0 & EDEM3 & 21.0 \\
\hline MIR-30E-5P & 27.0 & SOX4 & 21.0 \\
\hline MIR-30B & 27.0 & ZNF512B & 21.0 \\
\hline
\end{tabular}

of cartilage progenitor cells. In addition, miR-124A might play an important role in progression of OA through targeting $Q K I$ and MAP $1 B$. This study will provide important clues for understanding of the mechanisms of $\mathrm{OA}$ and the development of therapeutic targets for OA.

\section{Methods}

\section{Data acquisition and preprocessing}

The expression profile dataset GSE82107 was downloaded from the Gene Expression Omnibus (GEO; http://www.ncbi.nlm.nih.gov/geo/) database. The data was deposited into the following platform: GPL570 [HGU133_Plus_2] (Affymetrix Human Genome U133 Plus 2.0 Array) by Marieke et al., including 10 end-stage OA synovial biopsies from the Orthopedics department of the Radboud University Medical Center and 7 synovial biopsies from individuals without a joint disease [28]. The study protocols were approved by $\mathrm{CMO}$ region Arnhem-Nijmegen in Netherlands.
Table 4 The top 15 nodes ranked by degree values in the integrated network based on downregulated DEGs, transcription factor (TFs), and miRNA. The bold fonts indicate miRNA, the italic indicate TFs

\begin{tabular}{ll}
\hline Symbol & Degree \\
\hline QKI & Degree: 21.0 \\
SCD & Degree: 11.0 \\
NEGR1 & Degree: 11.0 \\
LEP & Degree: 11.0 \\
EPB41L4B & Degree: 10.0 \\
MIR-200B & Degree: 9.0 \\
MIR-200C & Degree: 9.0 \\
MIR-429 & Degree: 9.0 \\
MAP 1B & Degree: 9.0 \\
COL4A3 & Degree: 9.0 \\
PLIN1 & Degree: 9.0 \\
CEP350 & Degree: 8.0 \\
MTUS1 & Degree: 8.0 \\
MIR-124A & Degree: 7.0 \\
USP25 & Degree: 6.0 \\
\hline
\end{tabular}

\section{Identification of DEGs}

The data was normalized using RMA function of Affy package in $\mathrm{R}$ software (http://www.bioconductor.org/ packages/release/bioc/html/affy.html). Subsequently, DEGs between OA and control samples were identified using Bayes method of limma package in $\mathrm{R}$ software (http://www.bioconductor.org/packages/release/bioc/ html/limma.html). The threshold value of DEGs was set as $\mid \log$ fold change $(\mathrm{FC}) \mid>1.0$ and $P$ value $<0.05$.

\section{GO enrichment function and pathway analyses}

The GO function, KEGG pathway and Reactome pathway enrichment analyses were performed to reveal the DEGs involved in biological processes (BP) and pathways through an online database (www.biocloudservice.com). $P$ value $<0.05$ was used as the significant difference.

\section{Construction of the PPI network}

Proteins rarely act alone as their functions tend to be regulated. However, PPI network can provide a valuable framework to understand the functional organization of the proteome [29]. Therefore, the PPIs of DEGs was analyzed using STRING online tool (version 10.0). DEGs with combined score $>0.4$ were identified.

\section{Identification of miRNAs and TFs based on DEGs in PPI network}

Webgestalt was used to identify miRNAs that regulated DEGs in PPI network under the threshold value of gene count $\geq 4$ and adjusted $P$ values $<0.05$. In addition, TFs 


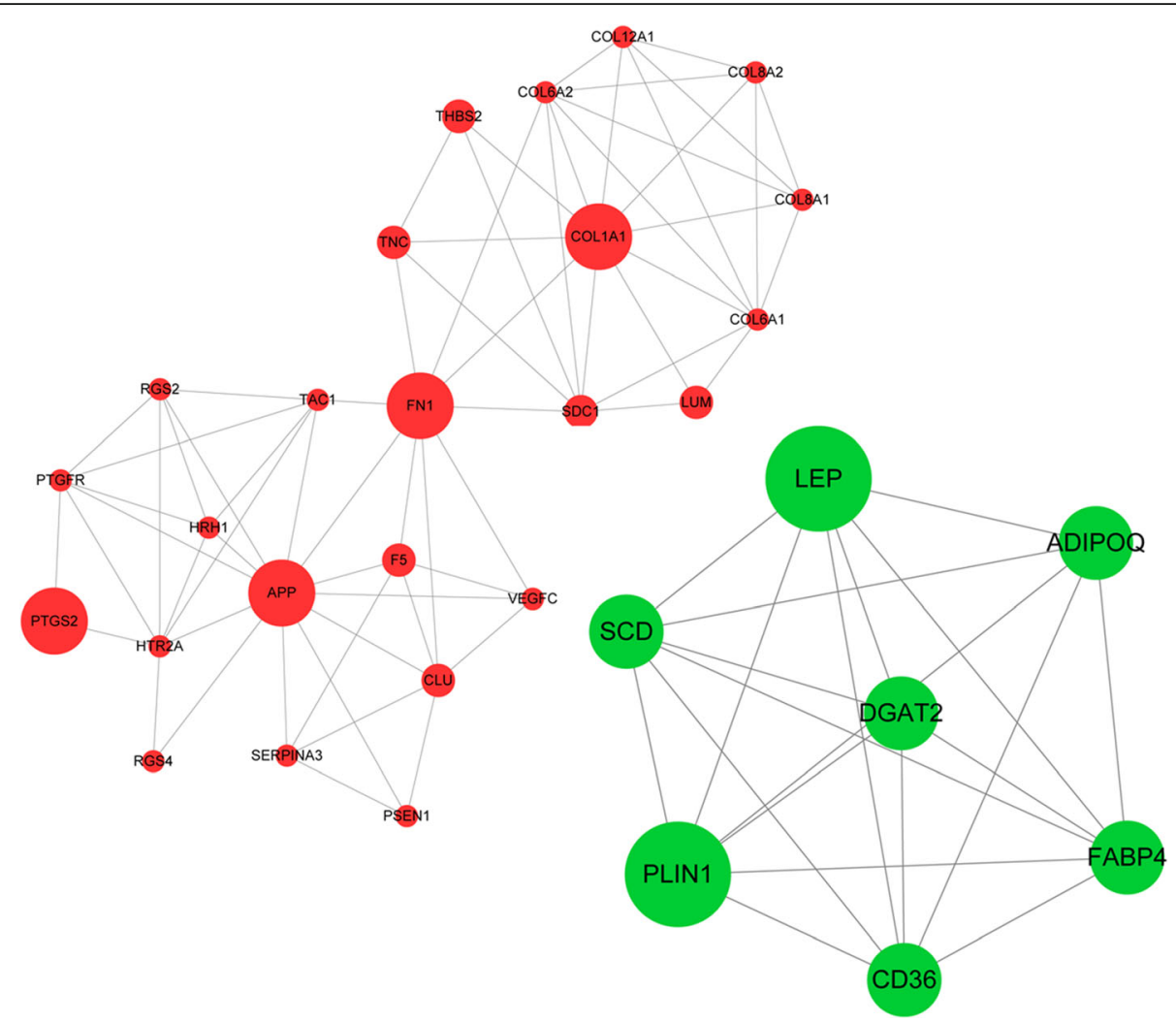

Fig. 4 The significant network module with the highest Mcode score (5.739) from the upregulated DEGs-TFs-miRNA integrated network and the module (Mcode score $=6.667$ ) from the downregulated DEGs-TFs-miRNA integrated network. Red nodes indicate upregulated DEGs, green nodes indicate downregulated DEGs

were screened from DEGs in the PPI network using ENCODE database [30].

The integrated network using DEGs in the PPI network, miRNA, and TFs

DEGs in PPI network and regulation relationships of miRNA-DEGs and TFs-DEGs were integrated to a network using Cytoscape (version 3.2.0, http://www.cytos cape.org/). As we known, the integrated network subjected to scale-free network. So the hub proteins were identified using connectivity degree analysis.

\section{Module analysis based on the integrated network}

MCODE was used to generate a sub-network for a list of significant network modules. The default threshold was set as following: Degree Cutoff: 2, Node Score Cutoff: 0.2, K-Core: 2, Max. Depth: 100. Subsequently, the GO function and KEGG pathway of significant network modules were enriched.

\section{Animal model of OA}

The male sprague dawley (SD) rats (weight $220 \pm 25 \mathrm{~g}$ and 8 week-old) purchased from SLAC Laboratory Animal Ltd. Co (Shanghai, China) were used to establish the animal model of OA. All procedures were conformed to the Institutional Animal Care and approved by Committee of Laboratory Animal Center of Nantong University. Rats were randomly divided into the control and model groups (30 rats each group). After acclimatization at the third day, rats in control group were given normal food without any other treatment. The left knee joints of rats in the model group were subjected to anterior cruciate ligament transaction (ACLT) according to the previous study [31], while the right knee joints of rats were served as sham control. Briefly, rats were anesthetized with pentobarbital sodium $(30 \mathrm{mg} / \mathrm{kg})$, then shaved and disinfected to expose knee joint. The patella was dislocated laterally and knee joint was buckled as much as possible. After anterior cruciate ligament (ACL) exposed, the ACL was transected with microscissors. Next, the joint surface was washed with sterile saline solution, and capsule and skin were sutured. In sham group, the operations were same except for ACLT. Penicillin was injected in a dose of 400,000 units per day for three consecutive days after surgery. Subsequently, rats were sacrificed and their joints were harvested at 8 weeks post-surgery. 


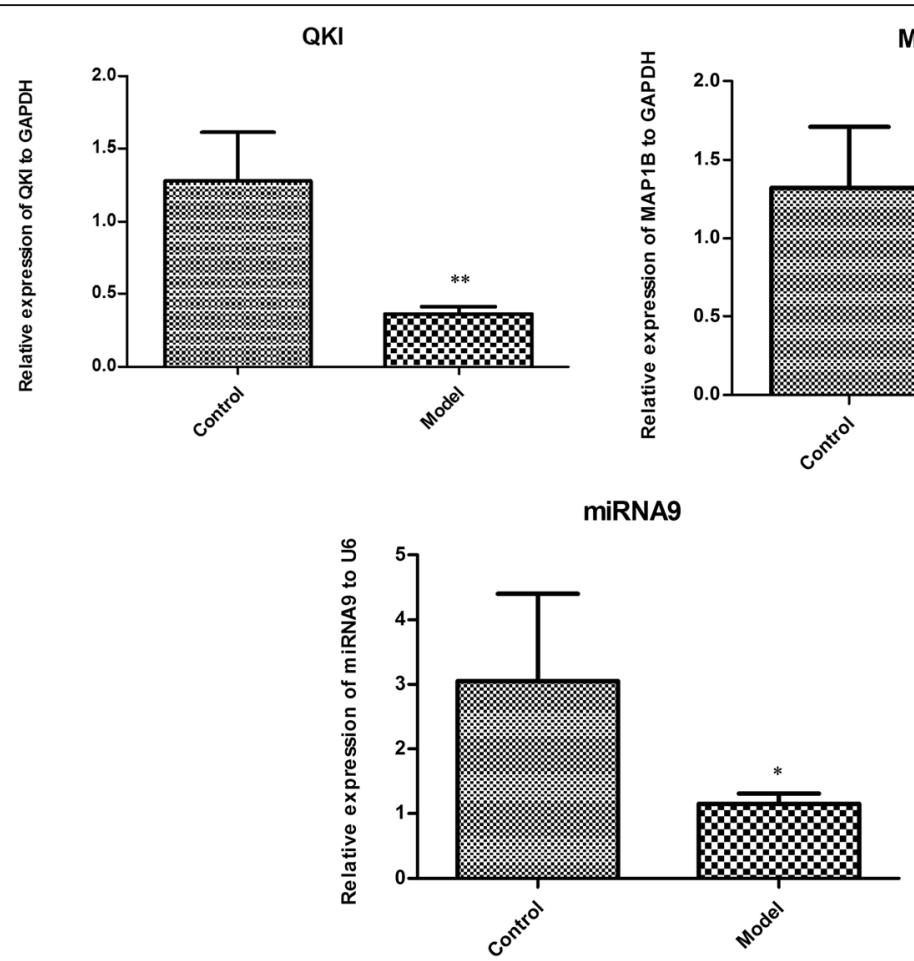

Fig. 5 The relative expressions of miR-9, QKI and MAP $1 B$. ${ }^{*} P<0.05$ indicates that there is significantly different between control and $O A$ samples. ${ }^{* *} P<0.01$

\section{The verification of qRT-PCR}

To confirm the above results, expression levels of QKI, MAP $1 B$ and miR-9 in OA samples (left knee joints) and sham control (right knee joints) were detected using qRT-PCR. Total RNA was extracted from synovial tissues of rats using RNAiso Plus reagent following the manufacturer's instructions (9109, TAKARA, Japan) under low temperature. Subsequently, the first strand cDNA was prepared from synovial tissues RNA using PrimeScript ${ }^{\text {tw }} \mathrm{RT}$ Master Mix according to the manufacturer's instructions (RR036A, TAKARA). Subsequently, the rat glyceraldehydes-3-phosphate dehydrogenase (GAPDH) was used as an internal reference. The primers used for QKI (Forward primer: AGTACCCCAT TGAACCCAGC; Reverse primer: TGTCTGGTAA AACAGTGGGGT) $M A P 1 B$ (Forward primer: ACGGTAGGGATTACAACG; Reverse primer: GACTCAGG GATGGACTCTT) and GAPDH (Forward primer: AGACAGCCGCATCTTCTTGT; Reverse primer: CTTGCCGTGGGTAGAGTCAT) were designed on rat sequences. The relative amounts of mRNAs were analyzed using Relative Expression Software Tool (REST). In addition, miR-9 (rno-miR-9a-5p-RT: GTCGTATCCA GTGCAGGGTCCGAGGTATTCGCACTGGATACG ACTCATAC; JH-rno-miR-9a-5p-Rf: GCGCGCTCTT TGGTTATCTAGCT) was also detected using qRTPCR. The rat U6 (forward primer: GCTTCGGCAG
CACATATACTAAAAT, reverse primer: CGCTTC ACGAATTTGCGTGTCAT) was used as an endogenous control. Relative gene expression was assessed by $2^{-\Delta \Delta C t}$ method.

\section{Statistical analysis}

The gene expression values in the $\mathrm{OA}$ and control groups were presented as mean \pm standard deviation $(\mathrm{X} \pm \mathrm{SD})$, and compared using unpaired Student's t-test by SPSS 22.0 (SPSS Inc., Chicago, IL, USA). $P<0.05$ was defined to be significantly different.

\section{Abbreviations}

DEGs: Differentially expressed genes; PPI: Protein-protein interaction; TFs: Transcription factor; GO: Gene Ontology; KEGG: Kyoto Encyclopedia of Genes and Genomes; OA: Osteoarthritis; miRNAs: MicroRNAs; TNF-a: Tumor necrosis factor a; GEO: Gene Expression Omnibus; BP: Biological processes

\section{Acknowledgements}

None.

\section{Authors' contributions}

Conception and design of the research: $\omega_{\text {; }}$ acquisition of data: $L$ J, XS; analysis and interpretation of data: XS; statistical analysis: HK; drafting the manuscript: $\mathrm{L}$; revision of manuscript for important intellectual content: HK. All authors read and approved the final manuscript.

Funding

None. 


\section{Availability of data and materials}

The datasets used and analysed during the current study are available from the corresponding author on reasonable request.

\section{Ethics approval and consent to participate}

Not applicable.

\section{Consent for publication}

Not applicable.

\section{Competing interests}

The authors declare that they have no competing interests.

Received: 27 November 2019 Accepted: 14 April 2020

Published online: 22 April 2020

\section{References}

1. Glynjones S, Palmer AJ, Agricola R, Price AJ, Vincent TL, Weinans $\mathrm{H}$, et al. Osteoarthritis. Lancet. 2015;386(9991):376-87.

2. Collaborators IP. Global, regional, and national incidence, prevalence, and years lived with disability for 310 diseases and injuries, 1990-2015: a systematic analysis for the global burden of disease study 2015. Lancet. 2016:388(10053):1545-602.

3. Harris KP, Driban JB, Sitler MR, Cattano NM, Balasubramanian E, Hootman $J M$. Tibiofemoral osteoarthritis after surgical or nonsurgical treatment of anterior cruciate ligament rupture: a systematic review. J Athl Train. 2017; 52(6):507-17.

4. Miyaki S, Asahara H. Macro view of microRNA function in osteoarthritis. Nat Rev Rheumatol. 2012;8(9):543-52.

5. Murata K, Yoshitomi H, Tanida S, Ishikawa M, Nishitani K, Ito H, et al. Plasma and synovial fluid microRNAs as potential biomarkers of rheumatoid arthritis and osteoarthritis. Arthritis Res Ther. 2010;12(3):R86.

6. Li Z-C, Han N, Li X, Li G, Liu Y-Z, Sun G-X, et al. Decreased expression of microRNA-130a correlates with TNF-a in the development of osteoarthritis. Int J Clin Exp Pathol. 2015;8(3):2555.

7. Kobayashi T, Lu J, Cobb BS, Rodda SJ, McMahon AP, Schipani E, et al. Dicerdependent pathways regulate chondrocyte proliferation and differentiation. Proc Natl Acad Sci. 2008;105(6):1949-54

8. Tuddenham L, Wheeler G, Ntounia-Fousara S, Waters J, Hajihosseini MK, Clark I, et al. The cartilage specific microRNA-140 targets histone deacetylase 4 in mouse cells. FEBS Lett. 2006;580(17):4214-7.

9. Miyaki S, Sato T, Inoue A, Otsuki S, Ito Y, Yokoyama S, et al. MicroRNA-140 plays dual roles in both cartilage development and homeostasis. Genes Dev. 2010;24(11):1173-85.

10. Fernandes JC, Martel-Pelletier J, Pelletier JP. The role of cytokines in osteoarthritis pathophysiology. Biorheology. 2002;39(1, 2):237-46.

11. Qi XJ, Wang JF, Wang GD, Xu Q, Sun HL. Pivotal role of microRNA-9 in osteosarcoma tumorigenesis and tumor progression. Genet Mol Res Gmr. 2016;15(1):gmr.15017318. https://doi.org/10.4238/gmr.15017318.

12. Jiang Y, Cai Y, Zhang W, Yin Z, Hu C, Tong T, et al. Human cartilage-derived progenitor cells from committed chondrocytes for efficient cartilage repair and regeneration. Stem Cells Transl Med. 2016;5(6):733-44.

13. Hayes AJ, Tudor D, Nowell MA, Caterson B, Hughes CE. Chondroitin sulfate Sulfation motifs as putative biomarkers for isolation of articular cartilage progenitor cells. J Histochem Cytochem Off J Histochem Soc 2008;56(2):125.

14. Jiang $Y$, Tuan RS, et al. Origin and function of cartilage stem/progenitor cells in osteoarthritis Nat Rev Rheumatol. 2015:11(4):206-12.

15. Topol A, Zhu S, Hartley BJ, English J, Hauberg ME, Readhead B, et al. Dysregulation of miRNA-9 in a subset of schizophrenia patient-derived neural progenitor cells. Cell Rep. 2016;15(5):1024.

16. Hu Y, Luo M, Ni N, Den Y, Xia J, Chen J, et al. Reciprocal actions of microRNA-9 and TLX in the proliferation and differentiation of retinal progenitor cells. Stem Cells Dev. 2014;23(22):2771-81.

17. Song J, Kim D, Chun CH, Jin EJ. MicroRNA-9 regulates survival of chondroblasts and cartilage integrity by targeting protogenin. Cell Communication Signaling. 2013;11(1):66

18. Nakamachi Y, Saegusa J, Kawano S. MicroRNA-124: a promising therapeutic agent for various human diseases, including rheumatoid arthritis. RNA DIS. 2017:4:76047028. https://doi.org/10.14800/rd.1252.
19. Miranda-Duarte A. Epigenetic mechanisms in osteoarthritis. In: Qian C, editor. Osteoarthritis: Progress Basic Res Treatment, IntechOpen. 2015. p. 15-38.

20. Mayer U, Springorum H-R, Grässel S. Influence of osteoarthritic cartilage explants on the expression of MIRNAS and predicted targets in cocultured chondrocytes and mesenchymal stem cells. Osteoarthr Cartil. 2016;24:S346

21. Yu X-M, Meng H-Y, Yuan X-L, Wang Y, Guo Q-Y, Peng J, et al. MicroRNAs' involvement in osteoarthritis and the prospects for treatments. Evid Based Complement Alternat Med. 2015;2015:1-13.

22. Vernet $C$, Artzt K. STAR, a gene family involved in signal transduction and activation of RNA. Trends Genet. 1997;13(12):479.

23. Li YH, Tavallaee G, Tokar T, Nakamura A, Sundararajan K, Weston A, et al. Identification of synovial fluid microRNA signature in knee osteoarthritis: differentiating early- and late-stage knee osteoarthritis. Osteoarthritis Cartilage. 2016:24(9):1577.

24. Gevorkian G, Gonzaleznoriega A, Acero G, Ordoñez J, Michalak C, Munguia $\mathrm{ME}$, et al. Amyloid-beta peptide binds to microtubule-associated protein 1B (MAP 1B). Neurochem Int. 2008;52(6):1030-6.

25. Tvorogova AA, Kovaleva AV, Saidova AA. Reorganization of actin cytoskeleton and microtubule array during the chondrogenesis of bovine MSCs. Annual Res Rev Biol. 2018;29(6):1-14.

26. Steinberg J, Grs R, Roumeliotis TI, Jayasuriya RL, Clark MJ, Brooks RA, et al. Integrative epigenomics, transcriptomics and proteomics of patient chondrocytes reveal genes and pathways involved in osteoarthritis. Sci Rep. 2017:7(1):8935

27. Zhao $L$, Ku L, Chen $Y$, Xia M, LoPresti P. Feng Y. QKI binds MAP $1 B$ mRNA and enhances MAP $1 \mathrm{~B}$ expression during oligodendrocyte development. Mol Biol Cell. 2006;17(10):4179-86.

28. Broeren MGA, Vries MD, Bennink MB, Lent PLEMV, Kraan PMVD, Koenders $\mathrm{MI}$, et al. Functional tissue analysis reveals successful cryopreservation of human osteoarthritic Synovium. PLoS One. 2016;11(11):e0167076.

29. De LRJ, Fontanillo C. Protein-protein interactions essentials: key concepts to building and analyzing interactome networks. PLoS Comput Biol. 2010;6(6): e1000807.

30. Consortium EP. Identification and analysis of functional elements in $1 \%$ of the human genome by the ENCODE pilot project. Nature. 2007; 447(7146):799.

31. Hayami T, Pickarski M, Zhuo Y, Wesolowski GA, Rodan GA, Duong LT. Characterization of articular cartilage and subchondral bone changes in the rat anterior cruciate ligament transection and meniscectomized models of osteoarthritis. Bone. 2006;38(2):234-43.

\section{Publisher's Note}

Springer Nature remains neutral with regard to jurisdictional claims in published maps and institutional affiliations.

Ready to submit your research? Choose BMC and benefit from:

- fast, convenient online submission

- thorough peer review by experienced researchers in your field

- rapid publication on acceptance

- support for research data, including large and complex data types

- gold Open Access which fosters wider collaboration and increased citations

- maximum visibility for your research: over $100 \mathrm{M}$ website views per year

At BMC, research is always in progress.

Learn more biomedcentral.com/submission 